\title{
Automatic Tool Selection System based on STEP_NC and IS013399 Standards
}

\author{
Hanae Zarkti ${ }^{1}$, Ahmed Rechia ${ }^{1}$ and Abdelilah El Mesbahi ${ }^{1}$ \\ ${ }^{1}$ Department of Mechanical Engineering, Faculty of Sciences and Technics, Tangier, Morocco. \\ zarktihanae@gmail.com, rechia@hotmail.com, elmesbahi_abdelilah@hotmail.com
}

\begin{abstract}
Computer Aided Process Planning (CAPP) is considered as an essential component of Computer Integrated manufacturing (CIM) environment and it is an important interface between Computer Aided Design (CAD) and Computer Aided Manufacturing (CAM). The purpose of the CAPP is to determine automatically the use of available resources, including machines, cutting inserts, holders, appropriate machining parameters such as cutting speed, feed rate, depth of cut, and generates automatic sequences of operations and instructions to convert a raw material into a required product, with good surface finish.

The contribution of this work in CAPP field is the development of an automatic tool selection system based on STEP_NC and ISO 13399 standards for turning and milling processes. The paper presents the result of study and analysis of principal system functionalities to be considered. The system consist of four principal modules: a Tool database, feature recognition module, cutting tool selection module and a process optimization module. Finally, based on functional analysis results, the paper present the development of tool database and data tool extraction module from ISO 13399 File using oriented object programing.
\end{abstract}

Keywords- CAPP; cutting tool selection; functional analysis; database; ISO13399; objected oriented programming;

\section{Introduction}

The ultimate goal of the factory of the future is to interconnect every step of the manufacturing process. Today, fast paced technology, sustainability, optimization and the need to meet customer demands have encouraged the transformation of the manufacturing industry, to become adaptive, fully connected and to adapt new standards that involve technical integration of systems across manufacturing processes.

One important stage when such an integration is required is the process planning. Actually, Computer Aided Process Planning (CAPP) represent an important interface between Computer Aided Design (CAD) and Computer Aided Manufacturing (CAM) and its integration is vital to the competitiveness of manufacturers.

Furthermore, the production cost of a manufacturing component depends upon cost of work-piece material, tooling cost, and recurring expenses. Thus, tool selection is considered as an important stage that contributes in the production cost of the manufacturing component. In the early 1980s, research 
Hanae Zarkti, Ahmed Rechia and Abdelilah El Mesbahi, Automatic Tool Selection System based on STEP_NC and ISO13399 Standards. Transactions on Machine Learning and Artificial Intelligence, Vol 5 No 4 August (2017);

p: $217-230$

work was undertaken in the area of computer-aided manufacturing and process planning systems have been developed to select a tool or a set of tools for a specific operation or a set of operations.

Xiaoping Ren, Zhanqiang Liu and Yi Wan developed a computer-based intelligent system for Automatic tool selection for different tool materials. The procedure of selection goes through several main steps: feature specification, machining type selection, cutting tool selection and optimum cutting conditions [1].

Jitender K. Rai, Daniel Brand, Mohammed Slama and Paul Xirouchakis developed a physical model called GA-MPO (genetic algorithm based milling parameters optimization system) in the multi-tool milling of prismatic parts [2].

C.G.Jensen, W.E.Red, J.Pi introduced automatic tool selection methods for five-axis curvatuve matched machining, based on cutter radius, cutter corner radius and cutter effective length [3].

Carpenter and Maropoulos [4] developed a flexible tool selection decision support system for milling operations, the system is called OPTIMUM (Optimized Planning of Tooling and Intelligent Machinability evaluation for Milling).It combine a knowledge based logic and statistical methods.

The authors implemented a module of machinability assessment that produces initial cutting conditions for a wide range of materials and tools. The system also proposes a new optimization criterion related to initial average chip thickness called harshness.

Roshan [5] has developed a graph based algorithm to find the optimal sequence for machining an entire setup that contains a set of features with precedence constraints.

The author presented four extensions to the basic algorithm:

feature level optimization, composite graph method, constrained graph method and sub-graph method.

In the first method, tool sequence graphs are built individually for each feature. In the second method, a composite tool sequence graph is generated for all features in a sibling level.

The constrained graph and subgraph approaches have been developed for situation where different features in the setup have distinct critical tools.

Mookhrejee and Bhattacharyya [6] developed an expert system Extool which automatically selects the turning tool/insert or milling insert, the material and geometry.

The machining time calculation function of the program requires the feed, cutting speed, depth of cut, length of job and the initial and required finished diameter of the job as input.

The outputs are cutting time and number of passes of the tool.

Edalew, Abdalla and Nash [7] developed a system for the selection of cutting tools. It's a dynamic programming based system that utilizes mathematical modules and heuristic data to determine and calculate cutting parameters and total component cost. The system contains the following modules:

the knowledge acquisition module, the knowledge base module, the inference engine, the user interface and the database. 
Arezoo, Ridgway and Al-Ahmari [8] developed a knowledge based system for selection of cutting tools and conditions of turning operations. It contains an inference engine, a user interface and explanation facility, a knowledge base and an optimization module for machining conditions.

Although recently, CAPP filed has received more attention from researchers and there have been considerable efforts in developing cutting tool selection systems there still a need for those systems to suit emerging standards of information exchange.

This is the need which the starting point of our research project stems from. Indeed, in the perspective of previous work on CAPP systems based on STEP_NC [9], [10], [11], [12], we aim to develop an automated optimized cutting tool selection system, integrated in a STEP_NC manufacturing chain, capable of assigning adequate tool to manufacture the part automatically and in an optimal manner.

The system comprises four modules: data tool extraction, feature recognition, Tool selection, and optimization modules.

The present work deals with development of data tool extraction module and the tool database.

\section{Functional Analysis}

We use functional analysis methodology to identify needs and to provide a clear representation of different functionalities of the system proposed.

\subsection{Identification of needs and System Functions}

The purpose of this design activity is to describe the functions of the system and its parts and indicates the mutual relations. Defining research needs in Fig.1 was the first stage of our work. We used "bêtes à corne" diagram to identify the need of the system. Need answers to three questions: 1 - who or what service makes the system? 2 - on what or who acts on the system? 3 - for what purpose, to do what?

The next step was to delimit system boundaries by defining existent interactions between the system and different features in its external environment. We used "Pieuvre" diagram to represent these interactions as two sort of functions: Principal functions (PF) and constraint functions (CF). Fig.2 represents connections created between the Tool selection System and the elements of its external environment, considering its using context and each stage of its lifecycle.

\subsection{Function decomposition and proposition of solutions}

After identification of functions, we develop a graphical representation FAST (Functional Analysis System Technique) Diagram showing the logical relationships between the functions of the system (Fig.3). 


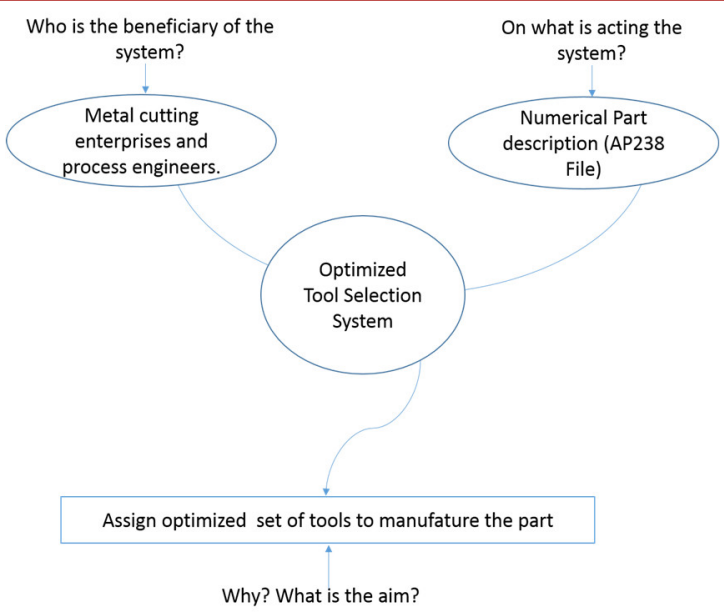

Figure.1. Identification of system need

The FAST diagram aims functions breakdown in subfunctions and eventually components breakdown which is governed by the following intuitive logic type questions like How? From left to right and why? Following the reverse, from right to left. It is used both to represent a known system as well to represent a new product based on its function.

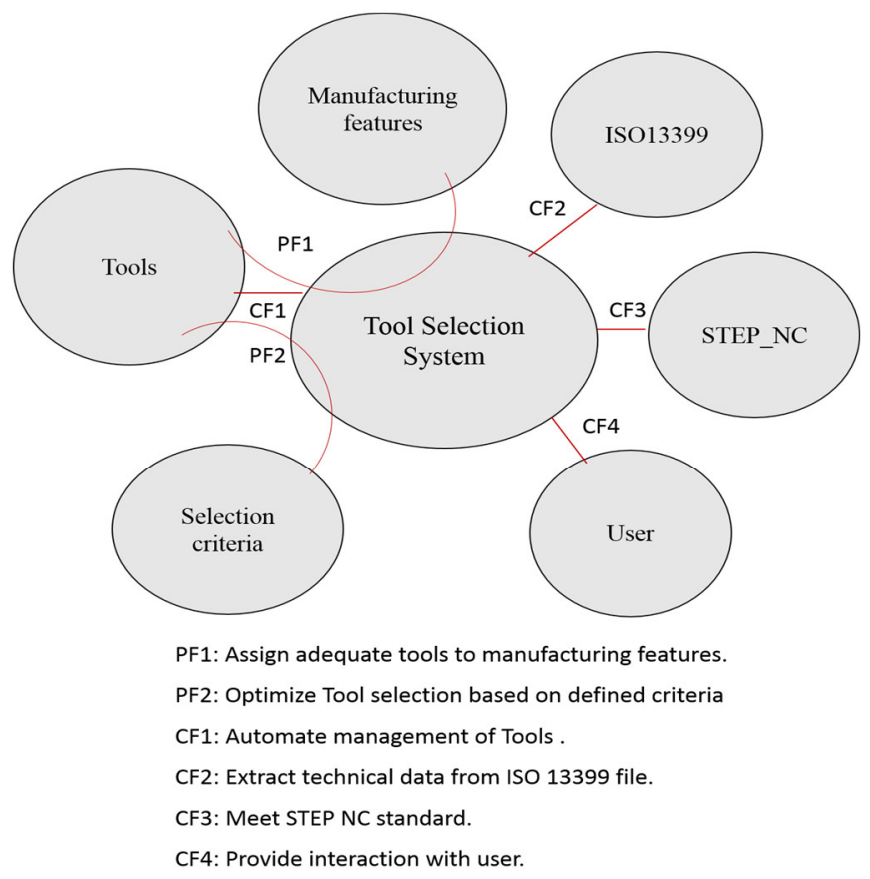

Figure.2. "pieuvre" diagram of the system 


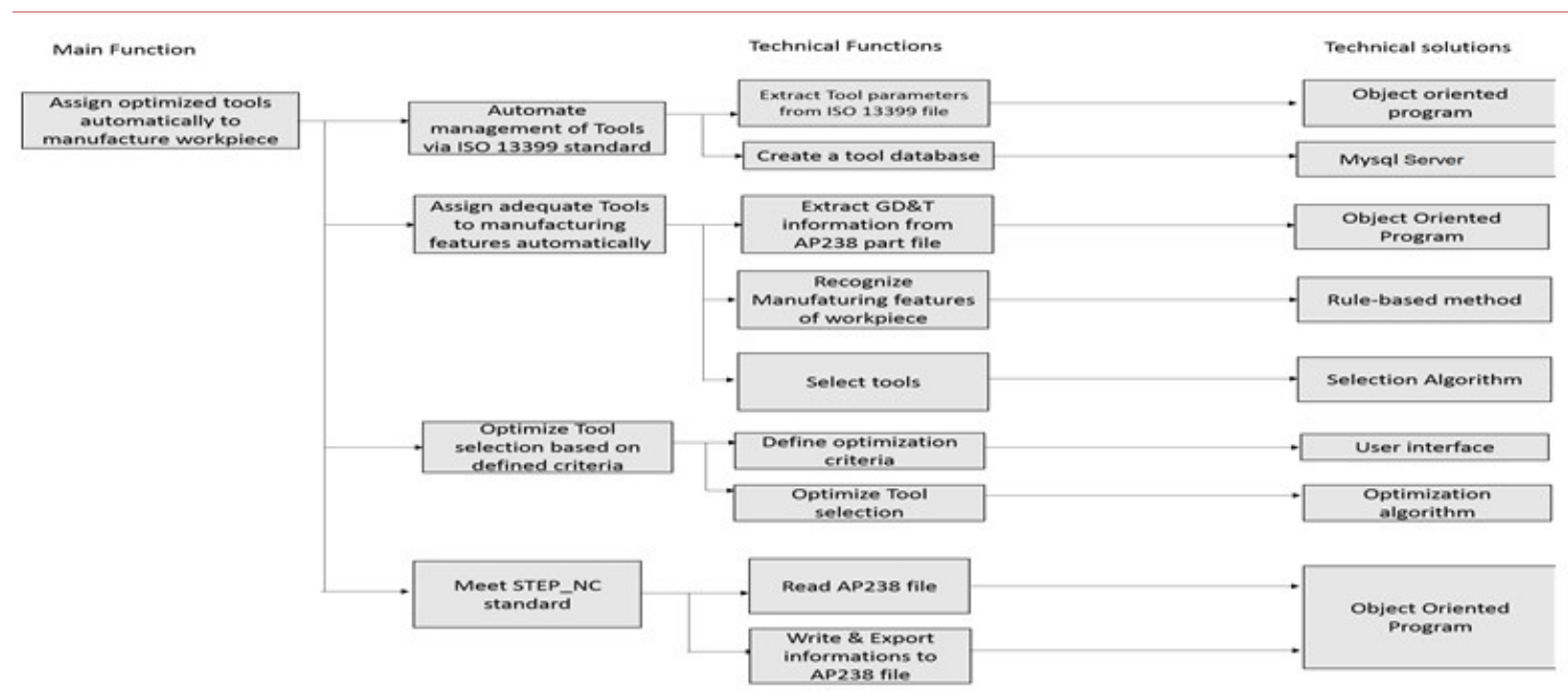

Figure .3 Functional Analysis System Technique diagram

\section{Automatic tool selection}

\subsection{Study of manual tool selection}

According to the manufacturer Sandvik Coromant [10], selection of cutters is done manually and goes through five principal stages:

1) Define the operation: The first is to identify the type of operation, then select the most suitable tool considering productivity, reliability and quality.

2) Define the material: Secondly material of the workpiece is defined according to ISO; Steel (P) - Stainless steel (M) - Cast iron (K) - Aluminum (N) - Heat resistant and titanium alloy $(\mathrm{S})$-Hardened material $(\mathrm{H})$.

3) Select the cutter concept and the cutter: Assess which concept is the most productive for the application, select the cutter and choose the type of mounting.

4) Select the insert: by choosing the insert geometry for the operation and selecting insert grade for optimum productivity.

5) Define the start values: Cutting speeds and feeds for different materials are given on the insert dispensers and in the tables. The values should be optimized according to machine and conditions.

In fact, the manual method presents some drawbacks; on one hand, it depends on the expertise of the user who must manually select parameters and criteria through the machining handbook for a given application, which is time consuming. On the other hand, the choice of tools provided by this method depends essentially on the operations to be performed and not on features to manufacture.

Thus, the aim of our project is to propose an automatic tool selection system in order to reduce Process planning time by using manufacturing feature approach instead of reasoning on operations.

Actually, we consider the part to manufacture as a group of manufacturing features (MF). Each MF has a number properties related to shape, dimensions, technology, tolerances etc. 


\subsection{Global System Architecture}

Based on the results of Functional analysis stage, we defined the global architecture of the automatic tool selection system.

Fig.4 shows flow chart of tool selection systems with inputs, outputs and relations between its modules: Data tool extraction module, recognition module, Selection module and optimization module.

The data tool extraction module is essential to the development of a solid tool database. Extraction procedure starts by uploading the ISO13399 file which contains information about tool, then these information are extracted and stored in the database.

Considering that the system is based on Step_NC standard, the process of tool selection starts by uploading the AP238 file of the Part. This numerical file contains necessary information to manufacture the part. It also comprises geometrical, topological and technical (material, tolerances...) definitions of the part to manufacture.

All of these technical definitions will be extracted using an object oriented program that allow also recognition of manufacturing features.

Then, based on information stored in Tool database, and using an appropriate tool selection algorithm, cutting tools are sorted and affected to Manufacturing features of the part.

A user interface allows planners to determine either to stop selection process and generate STEP_NC AP238 file for machining or to activate optimization module before generation.

In the second case, the user is invited to select optimization criteria and determine its preferences before starting optimization algorithm. Finally, optimal tools will be assigned to manufacturing features and the result is the numerical file STEP_NC AP238 which contains machining process information. 


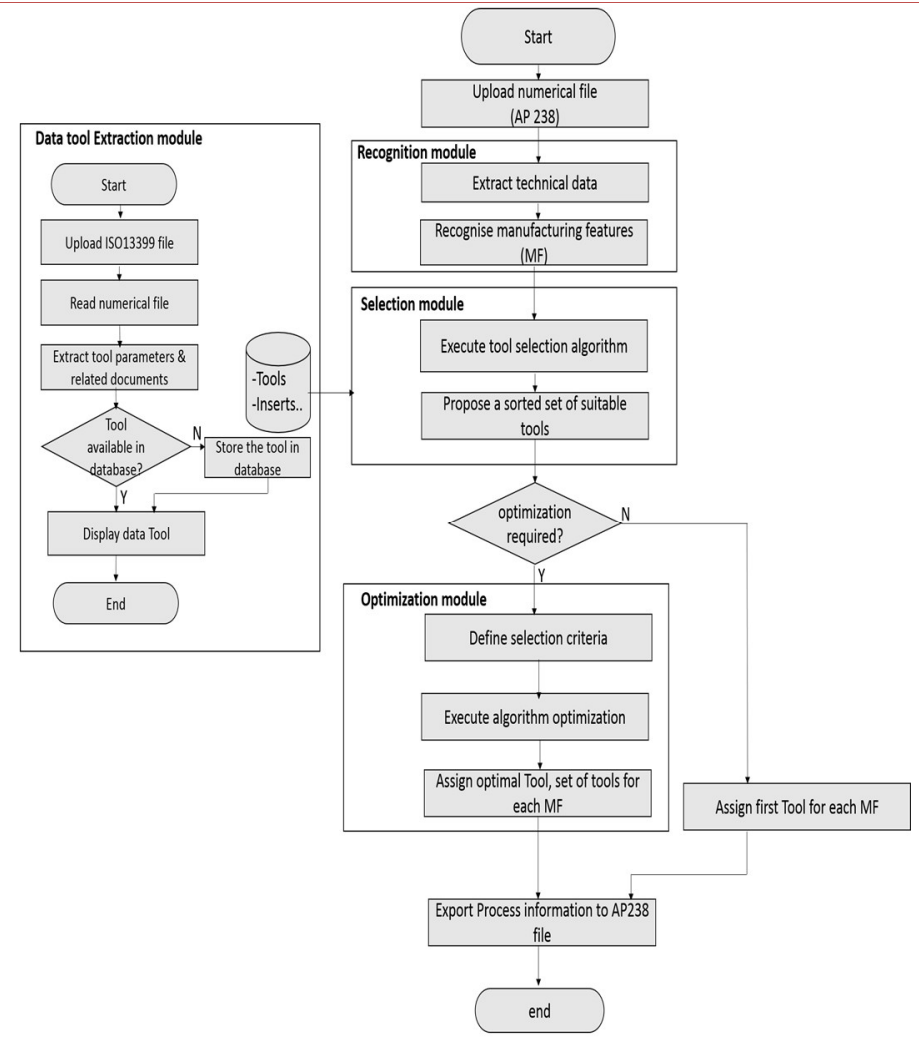

Figure 4. Tool representation in STEP_NC file

\section{Cutting tool information exchange}

Regarding the poor representation of tool in the older ISO $6983 \mathrm{G}$ and M code language, there is an increasing need for manufacturers to adapt a standard for cutting tool data representation. This urgent need emanates from the fact that the G\& M code describes only the path of the tool centre point with respect to machine axes. Consequently, even if the tool selection is done automatically with CAM systems, information about tool (geometry, properties...) will be lost when moving to the machining stage.

ISO 10303 AP238 (namely STEP_NC), and ISO 13399 are emerging standards on which the present work is based. Further information about these standards could be examined in the next section.

\subsection{Use of Step-Nc standard}

ISO 14649 or STEP-NC is a new standard developed for programming computerized numerical controllers (CNCS). The main development of STEP-NC was carried out in an international IMS/EU project with participants from Germany, France, Switzerland, South Korea, USA, and Sweden. The basic idea is to move toward a description of what to manufacture instead of the current description of how to manufacture. Description of cutting tool data for turning and milling are specified in ISO 14649 part 111[14] and ISO14649 part 121[15] respectively.

ISO 14649 defines a richer model for information transfer between CAD/CAM systems and computerized numerical control (CNC) machines than that of the older ISO 6983 " $G$ and M code" language. In ISO 6983, tools are defined with only their identifiers without any further information (e.g T3), while in a STEP_NC file, tools are defined in express language with values of properties required in machining (e.g cutting 
length, hand of tool, depth of cut ...) fig 5 is an excerpt of Step_Nc file of example CC2 in annex J in [16] where an end mill tool is defined with its properties.

\subsection{Use of IS013399 Standard}

ISO13399 includes the data representation of everything between the workpiece and the machine tool: Information about inserts (e.g. regular and irregular shaped replaceable cutting items), solid tools (e.g. solid drill and solid endmill), assembled tools (e.g. boring bars, indexable drills and indexable milling cutters), adaptors (e.g. milling arbor and chucks), components (e.g. shims, screws and clamps) or any combination of the above can be exchanged.

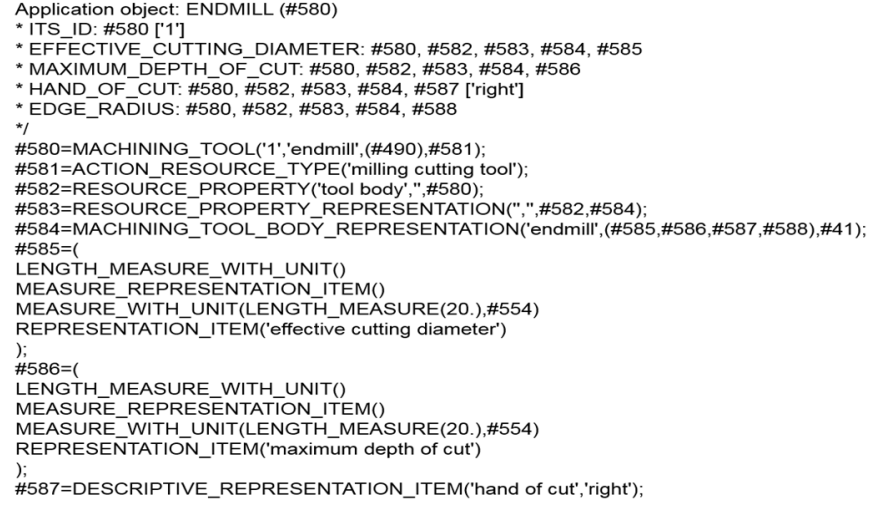

Figure 5. Tool representation in STEP _NC file

The cutting tool data described include, but are not limited to:

geometrical and dimensional data,

identification and designation data,

cutting material data,

and component connectivity.

The purposes of ISO 13399 and ISO 14649, in regards to tool information, are different. ISO 13399 is a standard for the description of cutting tool as products, while ISO 14649 only describes some simple tool requirements to be used by the CNC when deciding what cutting tool to use [17].

\section{Design of database}

\subsection{Study of cutting tool parameters and data collection}

One important step in designing tool database is to study
cutting parameters which are of great importance in selecting
cutting tools. Our main focus was on milling process. The result of study [18] shows three types of factors that influence tool selection:

\subsubsection{Parameters related to workpiece:}

Feature type: The type of feature is considered as a decisive parameter when selecting cutters. Suitable tools are selected by feature category. Indeed, machining features can be divided into two categories, 
simple features and complex features. Simple features are realized by using one machining operation, for example, a face and a shoulder can be performed by face milling and shoulder milling respectively. Complex features require at least two machining operations such as curved surfaces and freeform surfaces (features), which require roughing and profiling operations.

Surface roughness: Surface roughness is considered as a critical parameter to select tools and number of applications. For example, a feature having surface roughness of $0.8(\mathrm{Ra}=0.8)$ have to be machined in three applications, roughing, semi-finishing, and finishing application.

Material of the workpiece: Material of workpiece is a determinant factor for selecting the type of the cutter, the insert grade and the machining parameters. The manufacturer gives the designations of materials and their characteristics such as Specific cutting force and Brinell hardness. Depending on the insert grade and the type of material, the manufacturer gives the maximum chip thickness and the recommended cutting speed.

Dimensions of the feature: Dimensions of the feature represent an important parameter that lead the choice of milling tool. In fact, the depth of the feature is critical to determine the type of the cutter and the insert size, on the other hand the length of the feature represents an indicator of which diameter to choose.

\subsubsection{Parameters related to Cutting tool:}

Depth of cut (ap): In $\mathrm{mm}$ (axial) is what the tool removes in metal on the face from the workpiece. This is the distance the tool is set below the un-machined surface.

Tool material: Different machining applications require different cutting tool materials. The cutting tool material affect directly the cutting hardness, temperature stability and tool life.

Cutting width (ae): in $\mathrm{mm}$ (radial) is the width of the component engaged in cut by the diameter of the cutter. It is distance across the surface being machined or, if the tool diameter is smaller, that covered by the tool.

Entering angle: As regards cutting geometry in milling, the entering angle ( $\mathrm{kr}$ ), or the major cutting edge angle, of the cutter is the dominant factor affecting the cutting force direction and chip thickness. The choice of insert geometry has been simplified into three practical areas of varying cutting action effects: Light (L), general purpose $(\mathrm{M})$ and tough $(\mathrm{H})$ geometries.

Zn: The number of available cutter teeth in the tool varies considerably and is used to determine the table feed while the effective number of teeth (zc) is the number of effective teeth. The material, width of component, stability, power, surface finish influence how many teeth are suitable.

\subsubsection{Parameters related to machining process}

Cutting speed ( $v c$ ): in $\mathrm{m} / \mathrm{min}$ indicates the surface speed at which the cutting edge machines the workpiece. This is a tool oriented value and part of the cutting data which ensures that the operation is carried out efficiently and within the recommended scope of the tool material.

Spindle speed $(\mathrm{n})$ : in rpm is the number of revolutions the milling tool on the spindle makes per minute. This is a machine oriented value which is calculated from the recommended cutting speed value for an operation. 
Hanae Zarkti, Ahmed Rechia and Abdelilah El Mesbahi, Automatic Tool Selection System based on STEP_NC and

ISO13399 Standards. Transactions on Machine Learning and Artificial Intelligence, Vol 5 No 4 August (2017);

p: 217-230

Feed per minute (f): also known as the table feed, machine feed or feed speed, in $\mathrm{mm} / \mathrm{min}$ is the feed of the tool in relation to the workpiece in distance per time-unit related to feed per tooth and number of teeth in the cutter.

Maximum chip thickness (hex): in $\mathrm{mm}$ is the most important limitation indicator for a tool, for an actual operation. A cutting edge on a milling cutter has been designed and tested to have a recommended starting value and a minimum and maximum value.

\subsection{Data dictionnary and Elaboration of relational diagram}

The second step after definition of cutting parameters was to classify these parameters as properties of database entities or tables and to determine relationships between these entities via entity relationship modeling. The result is relational diagram of database depicted in figure 6 that we developed under MySQL workbench software. Inheritance relationships between classes were conceived based on tool and cutting item classification in ISO13399 part 2 [19] and ISO 13399 part 3[20] respectively. For example, a facemill derived from Mill class which derived from tool item class.

\subsection{Database implementation}

We choose to feed database automatically with data from The Sandvik Coromant [13] ISO 13399 catalogue for milling and turning tools.

For this purpose an object oriented program was created using c\# programming to extract technical data from ISO file and ensure data feed and update of database. More details on program functionalities are given in the following section.

\section{Extraction of Cutting Tool Data}

\subsection{ISO 13399 file structure}

An ISO 13399 exchange file contains an electronic representation of cutting tool data as defined by the information structure that can be exchanged by tooling applications.

ISO 13399 is defined as a Schema in the ISO 10303 Express language. The ISO 13399 schema develops a framework in which to define tooling properties, tooling assemblies, and relationship between tooling elements. There are no actual tooling properties in the ISO 13399 schema. Instead ISO 13399 develops a programming structure in which to embed ISO 13584, which is the Industrial automation systems and integration - Parts library (PLIB)[21]. 


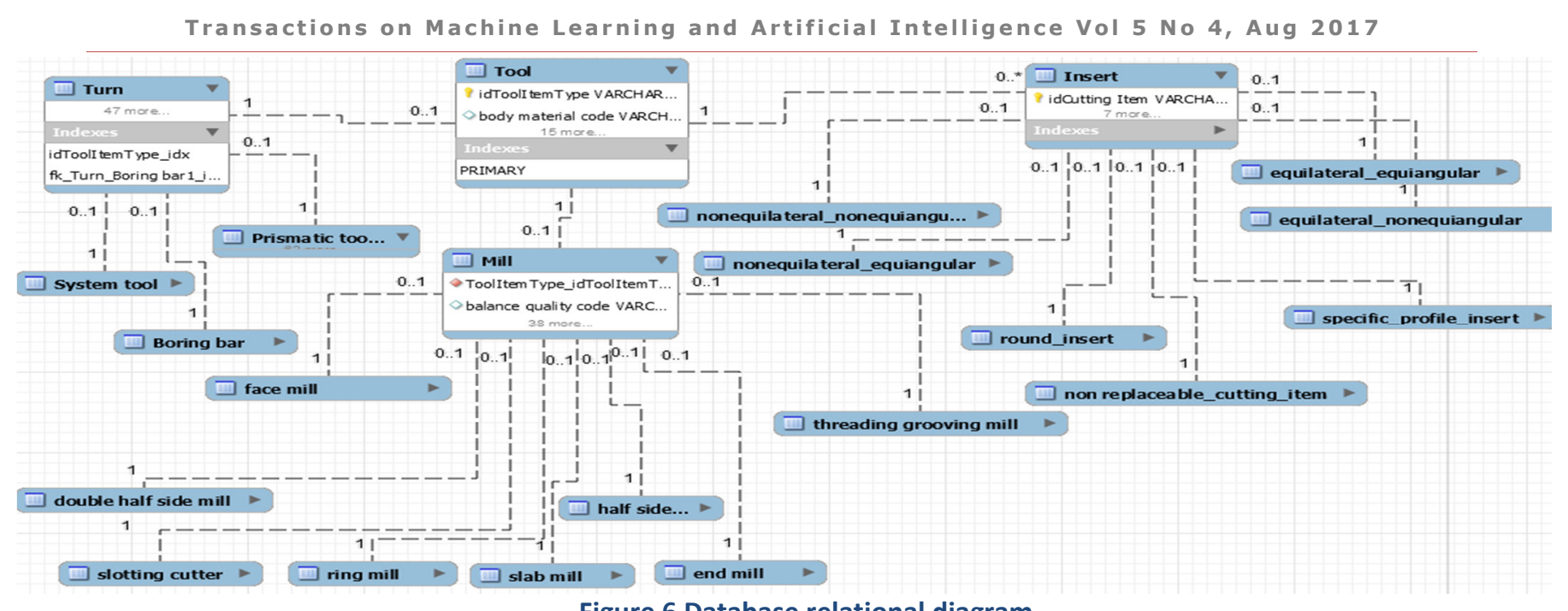

Figure 6 Database relational diagram

Figure 7 illustrate the main entities and relationship between them.

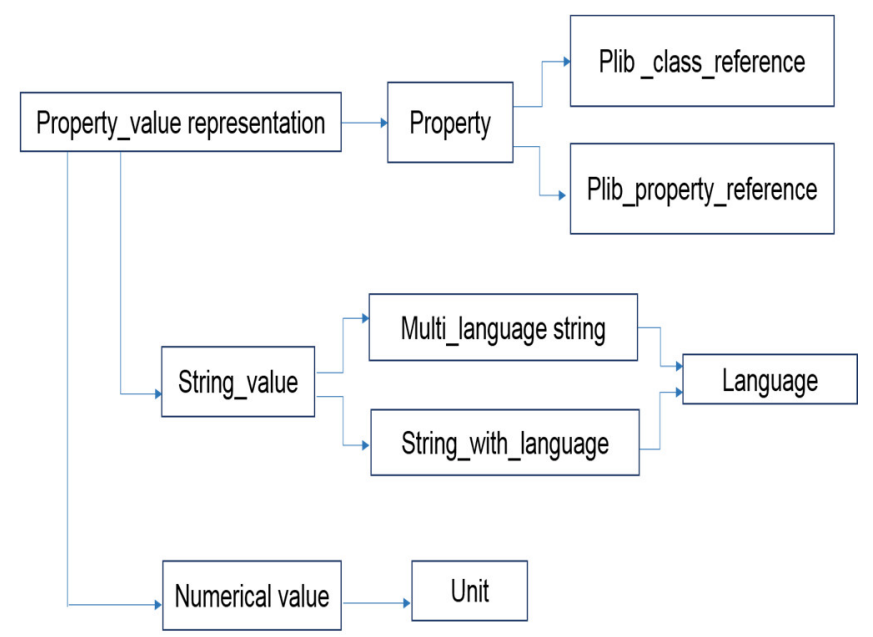

Figure 7 Sample Property Value Information Model Structure

A property_value_representation is used to define properties, based on a property and a value. Each property has a PLIB class and property reference. Definitions of entities linked to property_value representation class are as follow:

Property: A property is the definition of a particular quality. A property may reflect physics or arbitrary user defined measurements.

Plib_class_reference: A plib_class_reference designates a class in a library compliant to ISO 13584 (Parts Library).

Plib_property_reference: A plib_property_reference designates a property in a library compliant to ISO 13584.

String_value: A string_value represents a sequence of one or more alphanumeric characters. A string_value is a type of property_value.

Multi_language string: A multi_language_string represents text information, expressed in one or more languages, associated with object. 
String with language: A string_with_language represents text information in a specific language together with an identification of the language used.

Language: A language is a specification of the language in which information is given.

Numerical value: The specified_value specifies the property_value that qualifies the property_value_representation by a value_with_unit,.

Unit: specifies the unit in case the property value is with unit.

\subsection{Extraction of cutting tool data}

In this section we present the object oriented program that we developed using c\# programming. The extraction procedure and results are shown in figure 8. The purpose is to provide a fully integrated system that enables feed and modification of cutting tool database with recent tool information.

Tool data are extracted automatically from the ISO13399 file and stored in the database. After examination of the file data model, entities in figure 7 were mapped to c\# classes to be used in different part of the program. Extraction data tool scenario is as follow:

The user is invited to load the P21 file which is displayed in figure 8 (1). after a simple click, the extraction program is run and tool information are given in details in (2) the example shows a face milling cutter 'CoroMill 357' with different parameters. Also, names of correspondent inserts are displayed in a list box (3), and details could be reached after button click to run insert parameters extraction program and display results in (4). Finally, the user could add the new item to database or update information in (5).

\section{Conclusion}

The aim of this paper was to dress the architecture of our automatic optimized cutting tool selection system which is currently under development, based on functional analysis methodology. The paper dealt also with the development of the data tool extraction module and database that will be of great importance to the automation of cutting tool selection.

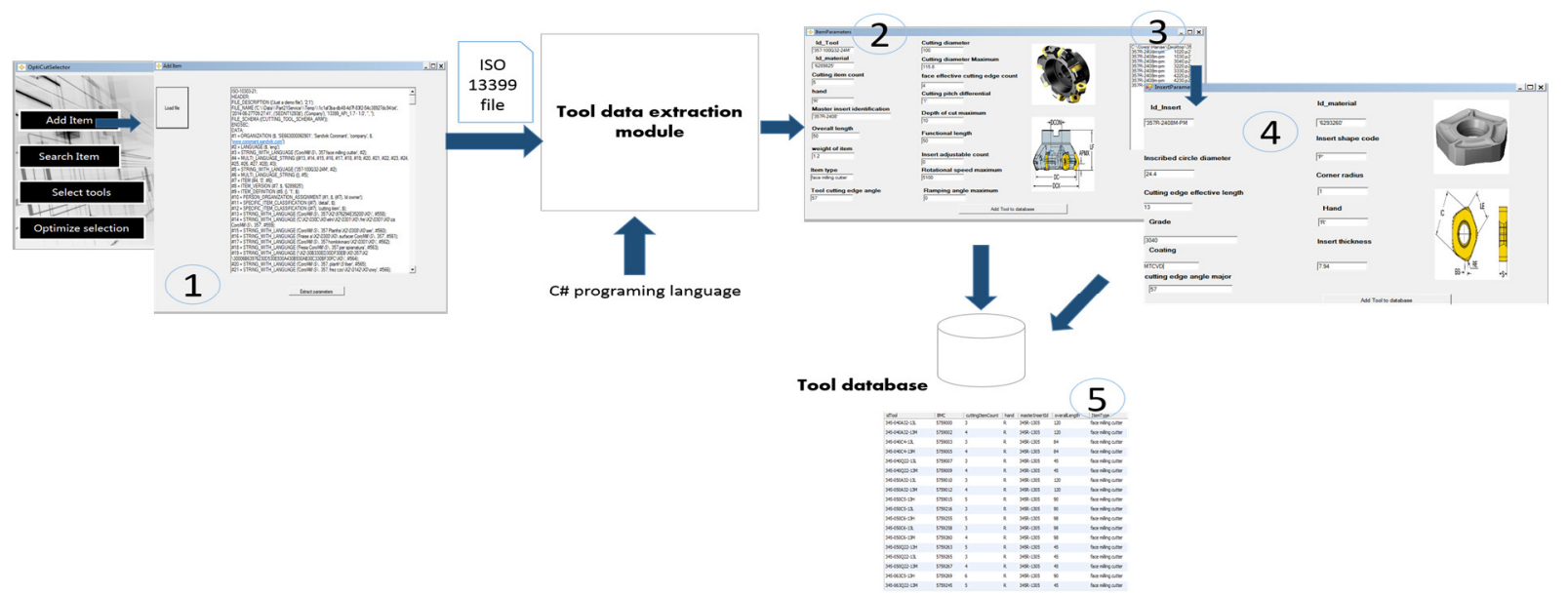

Figure 8. Cutting tool data extraction module 
The data tool extraction module was the automatic solution to feed and update database. Since tools manufacturers tends to adopt ISO13399 standard, we consider to update database with recent tool data to provide an effective way of tool selection. Development of feature recognition, tool selection, and optimization modules will be the object of future works.

\section{REFERENCES}

[1] X. P. Ren, Z. Q. Liu, Y. Wan, "A Computer-Based Intelligent System for Automatic Tool Selection", Materials Science Forum, Vol. 723, pp. 238-242, 2012.

[2] Jitender K. Rai , Daniel Brand, Mohammed Slama \& Paul Xirouchakis (2011) Optimal selection of cutting parameters in multi-tool milling operations using a genetic algorithm, International Journal of Production Research, 49:10, 3045-3068, DOI: 10.1080/00207540903382873.

[3] C. G. Jensen, W. E. Red, and J. Pi, "Tool selection for five-axis curvature matched machining," Comput. Aided D., vol. 34, pp. 251-266, Mar. 2002.

[4] I.D.Carpenter,P.G.Maropoulos, A flexible tool selection decision support system for milling operations, Journal of Materials Processing technology 107 (2000) 143-152.

[5] Roshan M.D'Souza, On setup level tool sequence selection for 2.5-D pocket machining, Robotics and Computer Integrated Manufacturing 22 (2006) 256-266.

[6] R.Mookherejee, B.Bhattacharyya, Development of an expert system for turning and rotating tool selection in a dynamic environment, Journal of Materials Processing Technology 113 (2001) 306-311. Figure 5.Tool data extraction module

[7] K.O. Edalew, H.S. Abdalla, R.J.Nash, A computer- based intelligent system form automatic tool selection, Materials and design 22 (2001) 337-351.

[8] B. Arezoo, K.Ridgway, A.M.A. Al-Ahmari, Selection of cutting tools and conditions of machining operations using an expert system, Computers in industry 42 (2000) 43-58.G. Eason, B. Noble, and I. N. Sneddon, "On certain integrals of Lipschitz-Hankel type involving products of Bessel functions," Phil. Trans. Roy. Soc. London, vol. A247, pp. 529-551, April 1955. (references)

[9] Abdelilah Elmesbahi, Ahmed Rechia, and Oussama Jaider, "optimized automated choice of cutting tool machining manufacturing features in milling process", proceeding of the 11th World Congress on Computational Mechanics (WCCM XI), Barcelone, 2014.

[10] Hanae Zarkti, Abdelilah El Mesbahi, Ahmed Rechia, Oussama Jaider. Towards an Automaticoptimized tool selection for milling process, based on data from Sandvik Coromant. X'eme Conf'erence Internationale : Conception et Production Intégrées, Dec 2015, Tanger, Morocco.

[11] Oussama Jaider, Abdelilah El Mesbahi, Ahmed Rechia, Hanae Zarkti. An automatic Feature-based tool selection approach for turning process based on data from Sandvik Coromant. Xème Conférence Internationale: Conception et Production Intégrées, Dec 2015, Tanger, Morocco. 
Hanae Zarkti, Ahmed Rechia and Abdelilah El Mesbahi, Automatic Tool Selection System based on STEP_NC and

ISO13399 Standards. Transactions on Machine Learning and Artificial Intelligence, Vol 5 No 4 August (2017);

p: $217-230$

[12] Oussama JAIDER, “Planification automatique des processus d'usinage des pièces de révolution, basée sur les normes STEP et STEP-NC", Thèse de doctorat, Faculté des Sciences et Techniques, Tanger, Maroc, 2017.

[13] www.sandvik.coromant.com/

[14] ISO 14649-111: Industrial automation systems and integration - Physical device control - Data model for computerized numerical controllers - Part 111: Tools for milling machines.

[15] ISO 14649-121: Industrial automation systems and integration - Physical device control -Data model for computerized numerical controllers - Part 121: Tools for turning machines.

[16] ISO 10303-238: Industrial automation systems and integration - Product data representation and exchange - Part 238: Application protocol: Application interpreted model for computerized numerical controllers.

[17] OLOF NYQVIST, Information Management for CuttingTools. Information Models and Ontologies, Doctoral thesis, Stockholm, Sweden 2008.

[18] Graham T. Smith, cutting tool technology: industrial handbook, Springer, 2008.

[19] ISO/TS 13399-2: Cutting tool data representation and exchange - Part 2: Reference dictionary for the cutting items.

[20] ISO/TS 13399-3: Cutting tool data representation and exchange - Part 3: Reference dictionary for the tool items.

[21] ISO/TC184/SC4 (2001a). Industrial Automation Systems and Integration - Parts Library - Part 1: Overview and fundamental principles. International Organization for Standardization. ISO 13584-1. 